\title{
Mixed Delay Constraints at Maximum Sum-Multiplexing Gain
}

\author{
Homa Nikbakht and Michèle Wigger \\ LTCI, Télécom ParisTech \\ 75013 Paris, France \\ \{homa.nikbakht, michele.wigger\}@telecom-paristech.fr
}

\author{
Shlomo Shamai (Shitz) \\ Technion \\ sshlomo@ee.technion.ac.il
}

\begin{abstract}
Coding schemes are proposed for Wyner's softhandoff model and for the sectorized hexagonal model when some of the messages are delay-sensitive and cannot profit from transmitter or receiver cooperation. For the soft-handoff network we also provide a converse. It matches the multiplexing-gain achieved by our scheme when the multiplexing gain of the delaysensitive messages is low or moderate or when the cooperation links have high capacities. In these cases, the sum-multiplexing gain is the same as if only delay-tolerant messages (which can profit from cooperation) were sent. A similar conclusion holds for the sectorized hexagonal model, when the capacities of the cooperation links are large.
\end{abstract}

\section{INTRODUCTION}

One of the major challenges of today's wireless communication networks is to design coding schemes for transmission of heterogeneous traffic types. Such a design, for example, has to account for the fact that delay-tolerant applications can profit from cooperation between transmitting or receiving terminals, but delay-sensitive applications cannot. Such mixed delay constraints in wireless networks have recently been studied in [1]-[5]. In this paper, we study the capacity of two cellular network models (Wyner's soft-handoff model [6], [7] and the hexagonal sectorized model) under mixed delay constraints. In a previous work, we have studied the capacity region of Wyner's soft-handoff network under mixed delay constraints when only the transmitters or only the receivers can cooperate [5]. We determined the multiplexing gain region in function of the capacities of the cooperation links. The results show that when delay-sensitive applications are of low rates, the total sum-rate is not decreased compared to a scenario where communication is only for delay-tolerant applications. In contrast, for high-rate delay-sensitive applications, this is not the case, and 1 bit of delay-sensitive rate comes at the expense of 2 bits delay-tolerant rate. Moreover, no positive delay-tolerant multiplexing gain is possible when one insists on sending at maximum delay-sensitive multiplexing gain.

As we show in this work, these (rather pessimistic) conclusions do not hold when both the transmitters and the receivers can cooperate. In fact, when the cooperation rates are sufficiently large, then by using sophisticated coding schemes it is possible to accommodate the largest possible multiplexing gain for delay-sensitive applications without decreasing the maximum sum-multiplexing gain. The stringent delay constraints thus do not harm the overall performance. For smaller

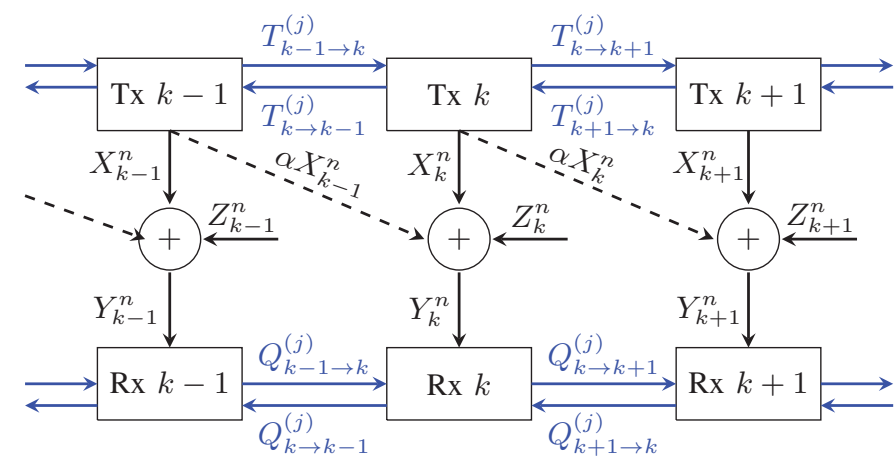

Fig. 1. System model

cooperation rates, this performance is achievable only when the delay-sensitive multiplexing gain is small. In this case, the behaviour is similar to when only transmitters or only receivers can cooperate.

\section{WYNER's Soft Hand-off NETWORK}

Consider the communication system as in Fig. 1 with $K$ interfering transmitter $(\mathrm{Tx})$ and receiver $(\mathrm{Rx})$ pairs $1, \ldots, K$. Transmitters and receivers are each equipped with a single antenna, and channel inputs and outputs are real valued. Interference is short-range so that the signal sent by $\mathrm{Tx} k$ is observed only by $\operatorname{Rx} k$ and $k+1$. As a result, the time- $t$ channel output at $\mathrm{Rx} k$ is

$$
Y_{k, t}=X_{k, t}+\alpha X_{k-1, t}+Z_{k, t},
$$

where $X_{k, t}$ and $X_{k-1, t}$ are the symbols sent by Tx $k$ and $k-1$ at time $t$, respectively; $\left\{Z_{k, t}\right\}$ are independent and identically distributed (i.i.d.) standard Gaussians for all $k$ and $t ; \alpha \neq 0$ is a fixed real number smaller than 1 ; and $X_{0, t}=0$ for all $t$.

Each Tx $k$ wishes to send a pair of independent source messages $\left(M_{k}^{(F)}, M_{k}^{(S)}\right)$ to Rx $k$. The "fast" source message $M_{k}^{(F)}$ is uniformly distributed over $\mathcal{M}_{k}^{(F)}:=\left\{1, \ldots,\left\lfloor 2^{n R_{k}^{(F)}}\right\rfloor\right\}$ and the "slow" source message $M_{k}^{(S)}$ over $\mathcal{M}_{k}^{(S)}:=$ $\left\{1, \ldots,\left\lfloor 2^{n R_{k}^{(S)}}\right\rfloor\right\}$. Here, $n, R_{k}^{(F)}$, and $R_{k}^{(S)}$ denote the blocklength and rates of transmission. Transmitters can cooperate with their immediate neighbours to exchange information about "slow" but not "fast" messages. In each cooperation round $j \in\left\{1, \ldots, \mathrm{D}_{t}\right\}$, Tx $k$ produces the two cooperation messages $T_{k \rightarrow k-1}^{(j)}$ and $T_{k \rightarrow k+1}^{(j)}$, where

$$
T_{k \rightarrow \tilde{k}}^{(j)}=\xi_{k \rightarrow \tilde{k}}^{(n)}\left(M_{k}^{(S)}, T_{k-1 \rightarrow k}^{(1)}, \ldots, T_{k-1 \rightarrow k}^{(j-1)},\right.
$$




$$
\left.T_{k+1 \rightarrow k}^{(1)}, \ldots, T_{k+1 \rightarrow k}^{(j-1)}\right)
$$

for some function $\xi_{k \rightarrow \tilde{k}}^{(n)}$ on appropriate domains. It sends these messages over the conferencing links to its left and right neighbours. The conferencing links are limited to rate $\pi$ :

$\sum_{j=1}^{\mathrm{D}_{t}} H\left(T_{k \rightarrow \tilde{k}}^{(j)}\right) \leq \pi \cdot n, \quad k \in\{1, \ldots, K\}, \tilde{k} \in\{k-1, k+1\}$.

Each Tx $(k)$ then computes its channel inputs as

$$
\begin{aligned}
X_{k}^{n}=f_{k}^{(n)}\left(M_{k}^{(F)}, M_{k}^{(S)}, T_{k-1 \rightarrow k}^{(1)}, \ldots, T_{k-1 \rightarrow k}^{\mathrm{D}_{t}},\right. \\
\left.T_{k+1 \rightarrow k}^{(1)}, \ldots, T_{k+1 \rightarrow k}^{\mathrm{D}_{t}}\right)
\end{aligned}
$$

for some function $f_{k}^{(n)}$ on appropriate domains that satisfies the average block-power constraint

$$
\frac{1}{n} \sum_{t=1}^{n} X_{k, t}^{2} \leq P, \quad \text { a.s. } \quad \forall k \in\{1, \ldots, K\} .
$$

Receivers decode in two phases. During the first fastdecoding phase, each $\mathrm{Rx} k$ decodes the "fast" source message $M_{k}^{(F)}$ based only on its own channel outputs $Y_{k}^{n}:=$ $\left(Y_{k, 1}, \ldots, Y_{k, n}\right)$. So, it produces:

$$
\hat{M}_{k}^{(F)}=g_{k}^{(n)}\left(Y_{k}^{n}\right)
$$

for some decoding function $g_{k}^{(n)}$ on appropriate domains.

In the subsequent slow-decoding phase, the receivers first communicate with their immediate neighbours over rate-limited cooperation links. In cooperation round $j \in$ $\left\{1,2, \ldots, \mathrm{D}_{r}\right\}, \mathrm{Rx} k$ sends the cooperation messages $Q_{k \rightarrow k-1}^{(j)}$ and $Q_{k \rightarrow k+1}^{(j)}$ to its neighbours $\operatorname{Rx}(k-1)$ and $\operatorname{Rx}(k+1)$, respectively. So, for $\tilde{k} \in\{k-1, k+1\}$ :

$$
\begin{aligned}
Q_{k \rightarrow \tilde{k}}^{(j)}=\psi_{k, \tilde{k}}^{(n)}\left(Y_{k}^{n}, Q_{k-1 \rightarrow k}^{(1)}, Q_{k+1 \rightarrow k}^{(1)}, \ldots,\right. & \\
& \left.Q_{k-1 \rightarrow k}^{(j-1)}, Q_{k+1 \rightarrow k}^{(j-1)}\right),
\end{aligned}
$$

for some encoding function $\psi_{k, \tilde{k}}^{(n)}$. The $\mathrm{D}_{r}$ messages sent over a conferencing link in each direction are rate-limited to $\pi$ :

$$
\sum_{j=1}^{\mathrm{D}_{r}} H\left(Q_{k \rightarrow \tilde{k}}^{(j)}\right) \leq \pi \cdot n, \quad k \in\{1, \ldots, K\}, \tilde{k} \in\{k, k+1\} .
$$

After this cooperation phase, each $\mathrm{Rx} k$ decodes its desired "slow" message as

$$
\left.\begin{array}{rl}
\hat{M}_{k}^{(S)}:=b_{k}^{(n)}\left(Y_{k}^{n}, Q_{k-1 \rightarrow k}^{(1)}, Q_{k+1 \rightarrow k}^{(1)},\right. & \\
\ldots, Q_{k-1 \rightarrow k}^{\left(\mathrm{D}_{r}\right)}, Q_{k+1 \rightarrow k}^{\left(\mathrm{D}_{r}\right)}
\end{array}\right)
$$

by means of a decoding function $b_{k}^{(n)}$.

Given cooperation prelog $\mu \geq 0$ and maximum delay $\mathrm{D}$, a pair $\left(\mathrm{S}^{(F)}, \mathrm{S}^{(S)}\right)$ is called achievable, if for each $K$ there exists a sequence of rate pairs $\left\{R_{K}^{(F)}(P), R_{K}^{(S)}(P)\right\}_{P>0}$ so that

$$
\mathrm{S}^{(F)}:=\varlimsup_{K \rightarrow \infty} \varlimsup_{P \rightarrow \infty} \frac{\sum_{k=1}^{K} R_{k}^{(F)}}{\frac{K}{2} \log (1+P)},
$$

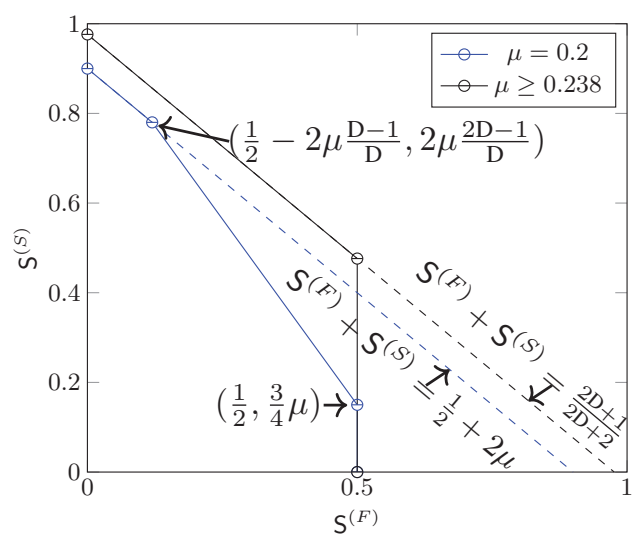

Fig. 2. Bounds on $\mathcal{S}^{\star}(\mu, \mathrm{D})$ for $\mathrm{D}=20$ and different values of $\mu$.

$$
\mathrm{S}^{(S)}:=\varlimsup_{K \rightarrow \infty} \varlimsup_{P \rightarrow \infty} \frac{\sum_{k=1}^{K} R_{k}^{(S)}}{\frac{K}{2} \log (1+P)},
$$

and so that for each rate pair $\left(R_{K}^{(F)}(P), R_{K}^{(S)}(P)\right)$ it is possible to find encoding, cooperation, and decoding functions with average power not exceeding $P$, total delay $\mathrm{D}_{t}+\mathrm{D}_{r} \leq \mathrm{D}$, and cooperation rate $\pi \leq \mu \cdot \frac{1}{2} \log P$, and so that the probability of decoding error

$$
P_{e}^{(n)}:=\operatorname{Pr}\left[\bigcup_{k \in\{1, \ldots, K\}}\left\{\hat{M}_{k}^{(F)} \neq M_{k}^{(F)} \text { or } \hat{M}_{k}^{(S)} \neq M_{k}^{(S)}\right\}\right]
$$

tends to 0 as $n \rightarrow \infty$. The closure of the set of all achievable $\left(\mathrm{S}^{(F)}, \mathrm{S}^{(S)}\right)$ is called multiplexing gain region and denoted $\mathcal{S}^{\star}(\mu, \mathrm{D})$.

The following is our main result in this section.

Theorem 1: The set $\mathcal{S}^{\star}(\mu, \mathrm{D})$ includes all nonnegative pairs $\left(\mathrm{S}^{(F)}, \mathrm{S}^{(S)}\right)$ satisfying

$$
\begin{aligned}
\mathrm{S}^{(F)} & \leq \frac{1}{2} \\
\mathrm{~S}^{(F)}+a \mathrm{~S}^{(S)} & \leq \frac{1}{2}+\frac{3 a}{4} \mu \\
\mathrm{S}^{(F)}+\mathrm{S}^{(S)} & \leq \min \left\{\frac{1}{2}+\frac{3}{4} \mu, \frac{2 \mathrm{D}+1}{2 \mathrm{D}+2}\right\},
\end{aligned}
$$

where $a:=\frac{8 \mathrm{D}-8}{13 \mathrm{D}-8}<1$.

Proof: See Section IV.

Proposition 1: Any pair $\left(\mathrm{S}^{(F)}, \mathrm{S}^{(S)}\right)$ in $\mathcal{S}^{\star}(\mu, \mathrm{D})$ satisfies

$$
\begin{aligned}
\mathrm{S}^{(F)} & \leq \frac{1}{2} \\
\mathrm{~S}^{(F)}+\mathrm{S}^{(S)} & \leq \min \left\{\frac{1}{2}+2 \mu, \frac{2 \mathrm{D}+1}{2 \mathrm{D}+2}\right\} .
\end{aligned}
$$

Proof: Follows from [8] and by a rate-transfer argument from "fast" to "slow" messages.

Fig. 2 depicts above bounds for two examples. The bounds coincide when $\mu$ is sufficiently large or $S^{(F)}$ sufficiently small.

Corollary 1: If

$$
\mu \geq \frac{4 \mathrm{D}}{3(2 \mathrm{D}+2)}
$$

then $\mathcal{S}^{\star}(\mu, \mathrm{D})$ is the set of pairs $\left(\mathrm{S}^{(F)}, \mathrm{S}^{(S)}\right)$ satisfying (15). 


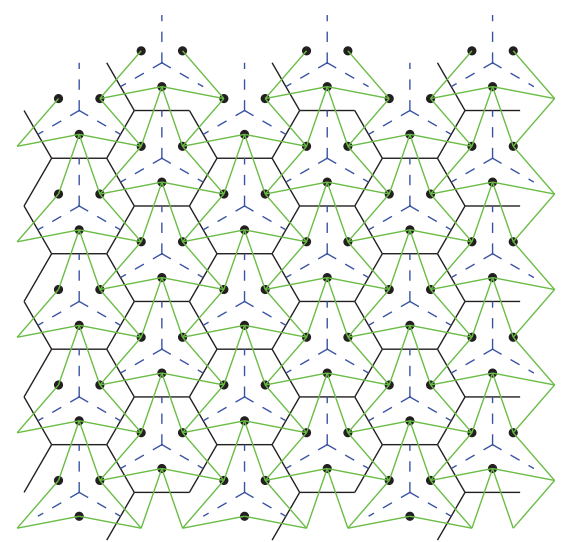

Fig. 3. Illustration of the sectorized hexagonal network. Black hexagonal regions depict the cells and the dashed blue lines determine their sectorization. Interfering sectors are connected by green lines.

If

$$
\mathrm{S}^{(F)} \leq \frac{1}{2}-2 \mu \frac{\mathrm{D}-1}{\mathrm{D}},
$$

then $\left(\mathrm{S}^{(F)}, \mathrm{S}^{(S)}\right) \in \mathcal{S}^{\star}(\mu, \mathrm{D})$ if, and only if, it satisfies (15b).

From Fig. 2 (and Corollary 1) we also see that when $\mu$ exceeds a threshold, then for any $\mathrm{S}^{(F)}$ the same maximum sum-multiplexing gain of $\min \left\{\frac{1}{2}+2 \mu, \frac{2 \mathrm{D}+1}{2 \mathrm{D}+2}\right\}$ is achievable as when only slow messages are transmitted. There is thus no penalty in sum-multiplexing gain by sending also "fast" messages. This is in contrast to the setup with only transmitter or receiver cooperation where a moderate or large multiplexing gain for "fast" messages causes a decrease in sum-multiplexing gain [5].

\section{Sectorized heXagonal Model}

In this model, the $N$ cells are hexagons and consist of three sectors. The BS of each cell is equipped with $3 M$ directional antennas, with $M$ antennas pointing to each sector. This allows avoiding interference between communications from different sectors in the same cell. For simplicity, and because the focus is on the multiplexing gain, we assume a single mobile user in each sector. The models with the cells, sectors, and the interference pattern, is depicted in Fig. 3. The small circles indicate mobile users, and the solid green lines indicate that the communication in two given sectors interfere.

For a given sector $k \in\{1, \ldots, 3 N\}$, let the set $\mathcal{I}_{k}$ contain the indices of the neighbouring sectors whose signals interfere with sector $k$. The time-t signal received at the $M$ receive antennas directing to sector $k$ can then be written as:

$$
Y_{k, t}=\mathrm{H}_{k, k} X_{k, t}+\sum_{\hat{k} \in \mathcal{I}_{k}} \mathrm{H}_{k, \hat{k}} X_{\hat{k}, t}+Z_{k, t},
$$

where $\left\{\mathrm{H}_{k, \hat{k}}\right\}$ denote the $M$-by- $M$ channel matrices.

As before, "fast" and "slow" messages are sent simultaneously over the network. Mobile transmitters in neighbouring sectors can cooperate "slow" messages during $\mathrm{D}_{t}$ rounds of cooperation. Receiving BSs decode their "fast" messages based solely on their own outputs and their "slow" messages based on these outputs and the cooperation signals that they exchanged with neighbouring $\mathrm{BSs}_{\text {over }} \mathrm{D}_{r}$ cooperation rounds.

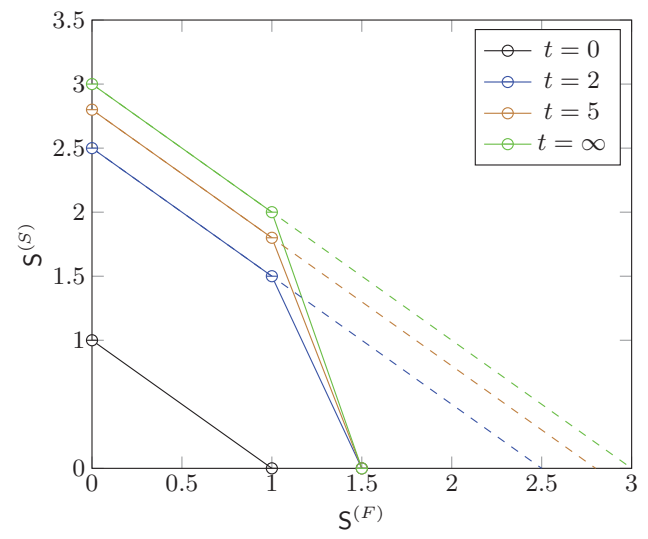

Fig. 4. Inner bound on multiplexing gain region for $M=3$ and different values of $t$.

So, BS $i$ produces as its guesses of the "fast" and "slow" messages:

$$
\hat{\mathbb{M}}_{i}^{(F)}=g_{i}{ }^{(n)}\left(\mathbb{Y}_{i}\right)
$$

and

$$
\hat{\mathbb{M}}_{i}^{(S)}=b_{i}^{(n)}\left(\mathbb{Y}_{i}, \mathbb{V}_{\text {to } i}^{j}\right)
$$

where $\mathbb{Y}_{i}:=\left\{\left(Y_{k, 1}, \ldots, Y_{k, n}\right)\right.$ : sector $k$ is in cell $\left.i\right\}$, $\mathbb{M}_{i}^{(F)}:=\left\{M_{k}^{(F)}:\right.$ sector $k$ is in cell $\left.i\right\}, \mathbb{M}_{i}^{(S)}:=\left\{M_{k}^{(S)}\right.$ : sector $k$ is in cell $i\}$ and $\mathbb{V}_{\text {to } i}^{j}$ denote all conferencing messages received at $\mathrm{BS} i$. The multiplexing gain region is defined analogously to the previous section, but for simplicity we assume that $\mu=1$.

Theorem 2: The multiplexing gain region includes all nonnegative pairs $\left(\mathrm{S}^{(F)}, \mathrm{S}^{(S)}\right)$ that satisfy

$$
\begin{aligned}
& \mathrm{S}^{(F)}+\mathrm{S}^{(S)} \leq \frac{M(3 t-1)}{3 t} \\
& \mathrm{~S}^{(F)}+c \mathrm{~S}^{(S)} \leq \frac{M}{2} .
\end{aligned}
$$

where $c:=\frac{t}{2(2 t-1)}<1$ and $t \in\left\{1, \ldots, \mathrm{D}_{r} / 2\right\}$.

Proof: See Section V.

This inner bound on the multiplexing gain region is illustrated in Figure 4. We notice that for small or moderate values of $\mathrm{S}^{(F)}$, the sum-multiplexing gain achieved by our scheme is the same as when only "slow" messages are sent.

\section{Schemes Achieving Theorem 1}

To achieve the performance in Theorem 1, four schemes need to be time-shared depending on the operating point and on the available cooperation prelog $\mu$.

\section{A. Scheme 1: Transmitting only "fast" messages}

In this scheme, every second transmitter is switched off, which decomposes the network into $\lceil K / 2\rceil$ non-interfering point-to-point links. A "fast" message of multiplexing gain 1 is transmitted over each of these point-to-point links. The scheme thus achieves multiplexing gain pair $\left(\mathrm{S}^{(F)}=1 / 2, \mathrm{~S}^{(S)}=0\right.$ ) without any requirement on the cooperation rate. 


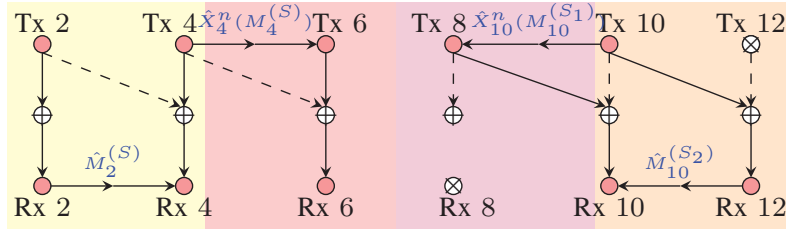

Fig. 5. Communicating "slow" messages in different groups of Scheme 4.

\section{B. Scheme 2: Transmitting only "slow" messages}

Use the scheme [8] to send only "slow" messages. It achieves multiplexing gain pair $\left(\mathrm{S}^{(F)}=0, \mathrm{~S}^{(S)}=\frac{2 \mathrm{D}+1}{2 \mathrm{D}+2}\right)$ with cooperation rate $\mu=\frac{\mathrm{D}}{4(\mathrm{D}+1)}$.

\section{Scheme3: Opportunistically sending "fast" messages}

Our third scheme is closely related to the second scheme. We notice that [8] splits the network into subnetworks by periodically silencing every $2 \mathrm{D}+2$-th transmitter. Also, the message of the left-most and the D+3-rd transmitters in each subnetwork do not participate in the transmitter cooperation and their decoding can be performed directly based on the corresponding channel output sequences. These messages can thus be sent as "fast" messages, whereas all other transmitted messages are "slow" messages. This scheme achieves multiplexing gain pair $\left(\mathrm{S}^{(F)}=\frac{2}{2 \mathrm{D}+2}, \mathrm{~S}^{(S)}=\frac{2 \mathrm{D}-1}{2 \mathrm{D}+2}\right)$ and requires cooperation rate $\mu=\frac{\mathrm{D}}{4(\mathrm{D}+1)}$, see [8].

\section{Scheme 4: Alternating "fast" and "slow" messages}

We again silence periodically every $2 \mathrm{D}+2$-th transmitter, so as to split the network into smaller subnetworks. In a given subnetwork, all odd transmitters $1,3,5, \ldots, 2 \mathrm{D}+1$ send a "fast" message with multiplexing gain 1 using a simple Gaussian codebook. They further precancel (up to noise level) the interference from their left-neighbours. To facilitate this, each even transmitter (which only sends a slow message as we explain shortly) quantizes and describes its input signal to its right neighbour over the cooperation link. All odd receivers $1,3,5, \ldots, 2 \mathrm{D}+1$ decode their desired "fast" message based on their own channel outputs using a simple point-to-point channel decoder. They also describe their decoded messages to their right-neighbours which then cancel the influence of the transmitted Gaussian codewords.

In the following, we describe the communication of "slow" messages, which is from even transmitters. Recall that all odd transmitters have precanceled a quantized version of their leftneighbours transmit signal. This implies that the signal sent by a given even Tx $k$ interferes at Rx $k+2$. Moreover, even receivers immediately cancel the interference of "fast" messages stemming from their left neighbours. The interference graph for "slow" messages thus has the form in Figure 5, where odd receivers are ignored. To describe communication of "slow" messages in more detail, we partition Tx/Rx pairs of each subnetwork into four groups.

Transmitters in $\mathcal{G}_{1}:=\left\{1, \ldots, D_{r}+1\right\}$ use Gaussian pointto-point codes of power $P$ to transmit their "slow" messages over the channel. Rx $k$ uses the cooperation messages received from its left neighbours $\mathrm{Rx} k-2$ (over two hops) and from $\mathrm{Rx} k-1$ to delete the interference

$$
\hat{Y}_{k}^{n}=Y_{k}^{n}-\alpha X_{k-1}^{n}\left(\hat{M}_{k-2}^{(S)}, \hat{M}_{k-1}^{(F)}\right),
$$

and it decodes the source message $M_{k}^{(S)}$ based on this difference. Then it describes its decoded message to $\mathrm{Rx} k+2$.

Transmitters in $\mathcal{G}_{2}:=\left\{\mathrm{D}_{r}+2, \ldots, \mathrm{D}_{r}+\mathrm{D}_{t}+1\right\}$ use dirtypaper coding to mitigate interference from the left. To this end, after receiving and reconstructing the quantization message $\hat{X}_{k}^{n}\left(M_{k-2}^{(S)}\right)$ sent by Tx $k-2$ (over two conferencing hops), Tx $k$ encodes its source message $M_{k}^{(S)}$ using a power $P$ dirtypaper code that eliminates the interference $\alpha \hat{X}_{k}^{n}\left(M_{k-2}^{(S)}\right)$. Then it sends the decoded message over the channel. Moreover, Tx $k$ quantizes its produced input $X_{k}^{n}\left(M_{k}^{(S)}\right)$ using a rate $(1 / 2) \log (1+P)$ quantizer and sends the resulting message to Tx $k+1$ and Tx $k+2$. Rx $k$ receives the decoded message $\hat{M}_{k-1}^{(F)}$ through the conferencing link to its left. Then it uses the received message to reconstruct $\alpha X_{k-1}^{n}\left(\hat{M}_{k-1}^{(F)}\right)$ and forms

$$
\hat{Y}_{k}^{n}=Y_{k}^{n}-\alpha X_{k-1}^{n}\left(\hat{M}_{k-1}^{(F)}\right) .
$$

It finally decodes message $M_{k}^{(S)}$ based on this difference.

Transmitters in $\mathcal{G}_{3}:=\left\{\mathrm{D}_{r}+\mathrm{D}_{t}+2, \ldots, \mathrm{D}_{r}+2 \mathrm{D}_{t}+2\right\}$ use dirty-paper coding to mitigate interference from the right. The desired communication path of a message $M_{k}^{(S)}$ is Tx $k \rightarrow$ Tx $k-2 \rightarrow \operatorname{Rx} k$. More specifically, Tx $k$ encodes $M_{k}^{(S)}$ and sends a quantized version $\hat{X}_{k}^{n}\left(M_{k}^{(S)}\right)$ to Tx $k-2$ which then transmits $X_{k-2}^{n}=\hat{X}_{k}^{n}\left(M_{k}^{(S)}\right)$ over the channel. Encoding is performed using dirty-paper coding where Tx $k$ precancels the interference of the signal $X_{k}^{n}$ it is sending over the channel. Receivers in $\mathcal{G}_{3}$ decode using standard dirty-paper decoding.

Transmitters in $\mathcal{G}_{4}:=\left\{\mathrm{D}_{r}+2 \mathrm{D}_{t}+3, \ldots, 2 \mathrm{D}_{r}+2 \mathrm{D}_{t}+1\right\}$, use a Gaussian point-to-point code of power $P$ to transmit their source messages $M_{k}^{(S)}$ over the channel through the path $\mathrm{Tx} k \rightarrow \mathrm{Rx} k+2 \rightarrow \mathrm{Rx} k$. Rx $k$ uses the cooperation messages received from its right neighbours $\mathrm{Rx} k+2$ (over two hops) and from $\mathrm{Rx} k+1$ to decode $M_{k}^{(S)}$.

In what we described, in some cases the last transmitter in $\mathcal{G}_{3}$ and the first transmitter in $\mathcal{G}_{4}$ are supposed to send the same message. In this case, we split this message into two independent parts, $M_{k}^{\left(S_{1}\right)}$ and $M_{k}^{\left(S_{2}\right)}$, and let each of the two transmitters send a different part. In the example in Fig. 5, Tx 8 sends $M_{10}^{\left(S_{1}\right)}$ and Tx 10 sends $M_{10}^{\left(S_{2}\right)}$.

The described scheme achieves multiplexing gain pair $\left(\mathrm{S}^{(F)}=\frac{1}{2}, \mathrm{~S}^{(S)}=\frac{\mathrm{D}}{2 \mathrm{D}+2}\right)$, and requires cooperation rate $\mu_{\max }=\frac{3 \mathrm{D}}{4(2 \mathrm{D}+2)}$.

\section{Schemes Achieving Theorem 2}

To achieve the performance in Theorem 2, three schemes need to be time-shared depending on the operating point.

\section{A. Scheme 1: Transmitting only "fast" messages}

Transmission is based on interference alignment [10], and cooperation links are completely ignored. This scheme achieves multiplexing gain pair $\left(\mathrm{S}^{(F)}=\frac{M}{2}, \mathrm{~S}^{(S)}=0\right)$. 


\section{B. Scheme 2: Transmitting only "slow" messages}

Use the scheme in [9] to send only "slow' messages. It achieves multiplexing gain pair $\left(\mathrm{S}^{(F)}=0, \mathrm{~S}^{(S)}=M \frac{3 t-1}{3 t}\right)$.

\section{Scheme 3: Alternating "fast" and "slow" messages}

The scheme is closely related to the second scheme. We notice that [9] defines master cells so that they build a regular grid of equilateral triangles where the three master cells forming each of the triangles lay $3 t$ cell-hops apart from each other for $t \in\left\{1, \ldots, \mathrm{D}_{r} / 2\right\}$. Layer- $1,2, \ldots, t$ cells are cells that are $1,2, \ldots, t$ hops away from master cells. The scheme in [9] deactivates users in layer-" $t$ " cells so as to split the network into non-interfereing clusters of sectors, see Fig. 6. In our scheme here, some users send "slow" messages and others send "fast" messages. Users sending "slow" messages use simple Guassian codebooks to send their messages. They quantize their inputs and send the quantization information to all their neighbours that send "fast" messages. The transmitters sending "fast" messages precancel the interference from "slow" messages. As we will see shortly, communication of "fast" messages is interfered only by "slow" messages, and since this interference is precanceled, receivers can decode their "fast" messages based on almost interference free signals. Decoded "fast" messages are sent to neighbouring receivers, which cancel the corresponding interference. In our scheme, all the three users of a master cell send "fast" messages. Users of layer-" 1 " cells that interfere users in the master cell send "slow" messages and the remaining users in this layer send "fast" message. Similarly, users of layer-"2" cells that interfere layer-" 1 " users send "slow" messages and the remaining layer"2" users send "fast" messages. A similar procedure is applied for layer-"3", layer-“4", ..., layer-"t-1" cells. In layer-"t" cells, all the active users send "slow" messages. Figure 6 shows the deactivated sectors in white, the sectors with "fast" messages in blue, and the sectors with "slow" messages in red.

This scheme achieves multiplexing gain pair $\left(\mathrm{S}^{(F)}=\right.$ $\left.\frac{M}{3}, \mathrm{~S}^{(S)}=M \frac{2 t-1}{3 t}\right)$.

\section{CONCLUSION}

We considered two cellular models and characterized the multiplexing gain region with transmitter and receiver cooperation when some source messages are subject to stringent delay constraints. In our previous work [5], increasing the rate of delay-sensitive messages by $\Delta$ required to decrease the rate of delay-tolerant messages approximately by $2 \Delta$. This penalty does not arise for the setup with transmitter and receiver cooperation that is considered for Wyner's soft-handoff model and the hexagonal sectorized model. In fact, when delaysensitive messages have moderate or small multiplexing gains, then the sum-multiplexing gain is not decreased compared to when only delay-tolerant messages are transmitted. For Wyner's soft-handoff model, this conclusion even holds for large multiplexing gains of "fast" messages when the cooperation rates are sufficiently large. The obtained results on transmitter and receiver cooperation may have interesting

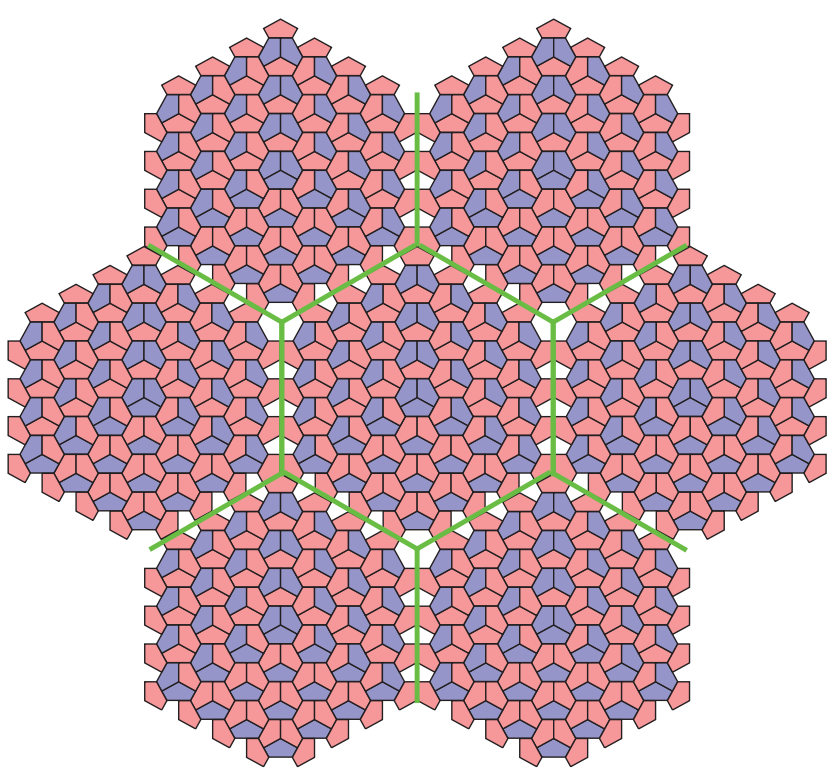

Fig. 6. Illustration of sector allocation for $t=4$. Users in blue sectors send "fast" messages and users in red send "slow" messages. White sectors indicate deactivated users, and thick lines indicate non-interfering clusters.

practical application due to the increase in different latency requirements.

\section{ACKNOWLEDGEMENT}

The work of H. Nikbakth and M. Wigger was supported by the ERC under grant agreement no. 715111. The work of S. Shamai has been supported by the European Union's Horizon 2020 Research And Innovation Programme, grant agreement no. 694630 .

\section{REFERENCES}

[1] K. M. Cohen, A. Steiner, and S. Shamai (Shitz) "The broadcast approach under mixed delay constraints," in Proc. IEEE ISIT 2012, Cambridge (MA), USA, July 1-6, 2012, pp. 209-213.

[2] R. Zhang, "Optimal dynamic resource allocation for multi-antenna broadcasting with heterogeneous delay-constrained traffic," IEEE J. of Sel. Topics in Signal Proc., vol. 2, no. 2, pp. 243-255, Apr. 2008.

[3] R. Zhang, J. Cioffi, and Y.-C. Liang, "MIMO broadcasting with delayconstrained and no-delay-constrained services," in Proc. IEEE ICC 2005, Seoul, South Korea, May 16-20, 2005, pp. 783-787.

[4] R. Kassab, O. Simeone and P. Popovski, "Coexistence of URLLC and eMBB services in the C-RAN uplink: an information-theoretic study," ArXiv:1804.06593.

[5] H. Nikbakht, M. Wigger and S. Shamai (Shitz), "Mixed delay constraints in Wyner's soft-handoff network," in Proc. IEEE ISIT 2018, Vail, CO, USA, June 17-22, 2018, pp. 1171-1175.

[6] A. D. Wyner, "Shannon-theoretic approach to a Gaussian cellular multiple-access channel," IEEE Trans. Inf. Theory, vol. 40, no. 6, pp. 1713-1727, Nov. 1994.

[7] S. V. Hanly and P. A. Whiting, "Information-theoretic capacity of multireceiver networks," Telecommunication Systems, vol. 1, pp. 1-42, 1993.

[8] M. Wigger, R. Timo, and S. Shamai (Shitz), "Conferencing in Wyners asymmetric interference network: effect of number of rounds," IEEE Trans. Inf. Theory, vol. 63, no. 2, pp. 1199-1226, Feb. 2017.

[9] S. Gelincik, M. Wigger, and L. Wang, "DoF in sectorized cellular systems with BS cooperation under a complexity constraint," online: https://perso.telecom-paristech.fr/wigger/ gelincik-ISWCS2018.pdf

[10] V. Ntranos, M. A. Maddah-Ali, and G. Caire, "Cellular interference alignment," IEEE Trans. Inf. Theory, vol. 61, no. 3, pp. 1194-1217, Mar. 2015. 